\title{
IMPORTANCE AND REPERCUSSIONS OF RENAL AND CARDIOVASCULAR PATHOLOGY ON STROKE IN YOUNG ADULTS: AN ANATOMOPATHOLOGIC STUDY OF 52 CLINICAL NECROPSIES
}

\author{
Gabriel Arismendi-Morillo, Mary Fernández-Abreu, José Cardozo-Duran, \\ Gustavo Vilchez-Barrios
}

Arismendi-Morillo G, Fernández-Abreu M, Cardozo-Duran J, Vilchez-Barrios G. Importance and repercussions of renal and cardiovascular pathology on stroke in young adults: an anatomopathologic study of 52 clinical necropsies. Clinics. 2008;63(1):15-20.

INTRODUCTION: Stroke in young adults has seldom been studied in a necropsy series. The objective of the present clinical necropsy-based investigation was to analyze stroke and its relationship with cardiovascular and renal pathology in young adults. MATERIALS AND METHODS: The protocols of 52 clinical necropsies with diagnoses of stroke in patients aged 18 - 49 years, performed between the years 1990-2006, were reviewed.

RESULTS: Hemorrhagic stroke was diagnosed in 36 patients (69.3\%), whereas the remaining 16 (30.7\%) had ischemic stroke. Hypertensive cardiopathy was evident in $88.4 \%$ of the cases. Chronic renal pathology, directly or indirectly related to hypertension, was observed in $55.7 \%$ of the patients. Ischemic stroke as a result of occlusive atherosclerotic disease was seen in $50 \%$ of cases. Cardiogenic emboli were found in $25 \%$ of the cadavers. Hemorrhagic stroke was associated with hypertension in $43 \%$ of the cases, with ruptured vascular malformations in $29 \%$, and coagulopathies in $17 \%$ of the cases. Hypertensive cardiopathy was present in patients with either ischemic or hemorrhagic stroke ( $81.2 \%$ and $91.6 \%$, respectively). The most frequently observed renal ailments were chronic pyelonephritis $(23 \%)$ and nephrosclerosis $(21.1 \%)$. These were associated with ischemic stroke in $43.7 \%$, and $12.5 \%$ of the cases, respectively, and with $13.8 \%$ and $25 \%$ of the hemorrhagic stroke cases.

DISCUSSION: Hypertensive cardiopathy, occlusive atherosclerotic disease, chronic pyelonephritis and nephrosclerosis are among the pathophysiologycal mechanisms that apparently and eventually interact to induce a significant number of cases of stroke in young adults. A chronic systemic inflammatory state appears to be an important related condition because it possibly constitutes an accelerant of the pathophysiologycal process.

KEYWORDS: Inflammation. Atherosclerosis. Chronic Kidney Disease. Hypertension. Hypertensive Cardiopathy.

\section{INTRODUCTION}

Stroke is a leading cause of death and disability worldwide. For this reason, stroke is of considerable socioeconomic importance ${ }^{1}$. According to the World Health Organi-

Universidad del Zulia - Instituto de Investigaciones Biológicas - Laboratorio de Microscopía Electrónica. Servicio de Anatomía Patológica y Nefrología del Hospital General del Sur "Dr. Pedro Iturbe" - Venezuela. gjam3000@yahoo.es

Received for publication on September 06, 2007. Accepted for publication on September 18, 2007. zation, stroke in young adults represents one of the 10 main causes of mortality and morbidity worldwide ${ }^{2}$. Human autopsy-based pathology studies remain the cornerstone of the current understanding of some aspects of stroke ${ }^{1}$. In spite of this, stroke in young adults has seldom been studied in necropsy series ${ }^{3}$. A review of the medical literature did not reveal any stroke- related neuropathological studies that scrutinize the concomitance of chronic cardiopathy and nephropathy in young adults. The aim of this investigation was to analyze this association, based on data obtained from clinical necropsies. 


\section{METHODS}

The protocols of 1,048 clinical necropsies performed at the Pathology Department of Hospital General del Sur "Dr. Pedro Iturbe" in Maracaibo, Venezuela from January 1990 - December 2006 were reviewed. Patients 18 - 49 years of age with a final diagnosis of stroke accounted for 52 of the autopsy cases. General pathological findings such as hypertensive cardiopathy, acute and chronic ischemic cardiopathy, nephrosclerosis, and chronic kidney disease, as well as the neuropathological aspects of stroke and cause of death were analyzed.

Hypertensive cardiopathy, defined as gross left ventricular hypertrophy in absence of specific causes, and histopathological observation of hyperthropic myocardial fibers with voluminous nuclei, was considered as the direct resultant of intravitam hypertension. For diagnoses of diverse cardiovascular pathologies, classical pathological criteria were used.

Diagnostic pathological criteria for nephrosclerosis included arteriolar hyalinization, fibroblastic hyperplasia of medium caliber arteries and global glomerulosclerosis associated with focal tubular atrophy and interstitial fibrosis. For diagnoses of diverse renal pathologies, classical pathological criteria were applied.

Diagnosis of hypertensive stroke was based on the presence of a hemorrhage in the basal ganglia, internal capsule, diencephalic structures, cerebellar hemispheres or brain stem, or the presence of lacunar infarctions in the basal ganglia, internal capsule and diencephalon.

To establish the prevalence of stroke in people 18 to 49 years old, statistical charts from the Clinical Histories Department were reviewed.

The SPSS-13.0 package was used for statistical analysis. Data are presented as means + standard deviation. Significance testing was performed by Student's $t$ test with
Yates' Correction and chi-square test. A $p$-value $<0.05$ was considered statistically significant in both tests.

\section{RESULTS}

Stroke in cadavers 18 to 49 years of age was present in $52(4.96 \%)$ of the 1,048 necropsies performed in this study. Revision of the statistical records of the Department of Clinical Histories allowed us to establish that stroke in people 18 to 49 years old corresponded to 891 cases $(21 \%)$ with a general mortality rate of $36 \%$, based on a total of 4,275 patients with cerebrovascular disease.

Male cadavers accounted for $59.6 \%$ of the cases. Hypertensive cardiopathy was observed in 46 cadavers (88.4\%), while 29 cases (55.7\%) showed at least one of the following chronic nephropathies: pyelonephritis, nephrosclerosis, glomerulonephritis, lupus nephropathy or renal atrophy. These entities were considered to be directly or indirectly related to arterial hypertension. Either chronic pyelonephritis or nephrosclerosis were evident in $44 \%$ of the cases (23\% and $21 \%$, respectively). Hypertensive stroke accounted for 15 cadavers (41.6\%), 12 (80\%) of which presented with at least one type of arterial hypertension-related chronic nephropathy. Table 1 shows the general data related by age and the main cardiopathies and nephropathies for stroke in young people.

\section{Ischemic Stroke}

Ischemic stroke was diagnosed in 16 cadavers $(30.7 \%)$. Ischemic stroke cases revealed multiple infarcts in five instances $(31.2 \%)$, hemispheric infarct in five patients $(31.2 \%)$, basal ganglia infarct in four $(25 \%)$, one in the pons $(6.2 \%)$ and one in the corpus callosum (6.2\%). Figure 1 displays the causes of ischemic stroke. Chronic pyelonephritis was present in seven $(43.7 \%)$ cadavers with

Table 1 - General Data related by Age, Principal Cardiopathies and Nephropathies for Stroke in Young People.

\begin{tabular}{|c|c|c|c|c|c|c|c|}
\hline & Ischemic Stroke & $\%$ & Hemorrhagic Stroke & $\%$ & Total & $\%$ & $p$ \\
\hline No. Cases & 16 & 30.7 & 36 & 69.3 & 52 & & \\
\hline Age (years) & $40.87 \pm 9.83$ & & $36.86 \pm 9.19$ & & & & $\mathrm{NS}^{\mathrm{a}}$ \\
\hline \multicolumn{8}{|l|}{ Cardiopathies } \\
\hline Hypertensive Cardiopathy & 13 & 81.2 & 33 & 91.6 & 46 & 88.4 & $\mathrm{NS}^{\mathrm{b}}$ \\
\hline Chronic Ischemic Cardiopathy & 3 & 18.7 & 2 & 5.5 & 5 & 9.6 & $\mathrm{NS}^{\mathrm{b}}$ \\
\hline $\begin{array}{l}\text { Previous Acute Myocardial Infarction } \\
\text { Nephropathies }\end{array}$ & 5 & 31.2 & 1 & 2.7 & 6 & 11.5 & $0.01^{\mathrm{b}}$ \\
\hline Nephrosclerosis & 2 & 12.5 & 9 & 25 & 11 & 21.1 & $\mathrm{NS}^{\mathrm{b}}$ \\
\hline Chronic Pyelonephritis & 7 & 43.7 & 5 & 13.8 & 12 & 23 & $0,04^{\mathrm{b}}$ \\
\hline Tubulointerstitial Nephropathy & 1 & 6.2 & 2 & 5.5 & 3 & 5.7 & $\mathrm{NS}^{\mathrm{b}}$ \\
\hline Other & 4 & 25 & 6 & 16.6 & 10 & 19.2 & $\mathrm{NS}^{\mathrm{b}}$ \\
\hline
\end{tabular}

${ }^{\mathrm{a}} t$ Student test with Yates' Correction; ${ }^{\mathrm{b}}$ Chi-square test; NS not significant. 


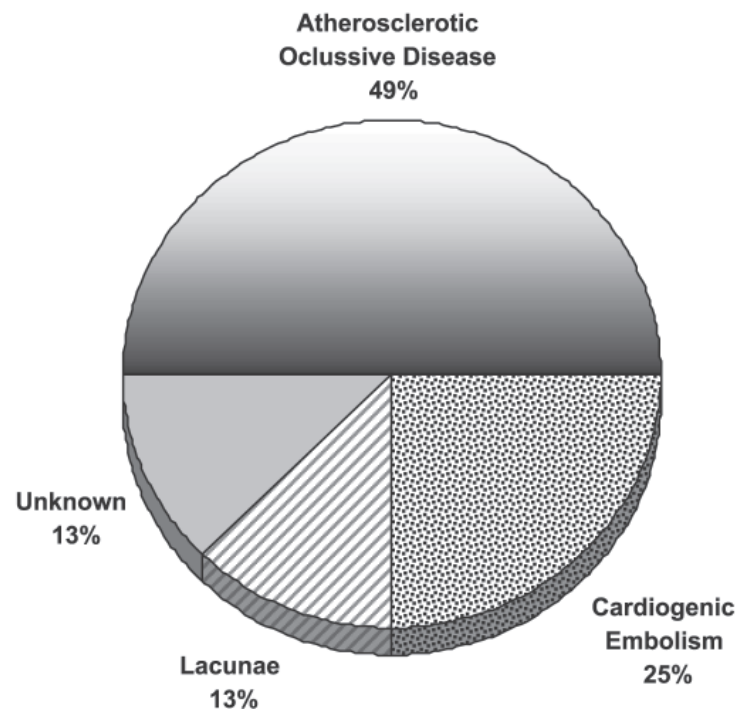

Figure 1 - Causes of Ischemic Stroke in Young People.

ischemic stroke. Nephrosclerosis was diagnosed in two patients $(12.5 \%)$. Causes of death in the ischemic stroke group were as follows: pulmonary causes in five cases (31.2\%), cardiovascular in four (25\%), sepsis in four $(25 \%)$ and other causes in three $(18.7 \%)$.

\section{Hemorrhagic Stroke}

Hemorrhagic stroke was present in 36 cadavers and represented $69.3 \%$ of the cases. Figure 2 displays the causes of hemorrhagic stroke. Hemorrhagic stroke appeared as an intraparenchymal hematoma in 18 cases (50\%); of these, $14(78 \%)$ cases were hypertensive in nature. In ten instances, the lesion was located in the basal ganglia, in three of the patients the pons was involved and in one case the

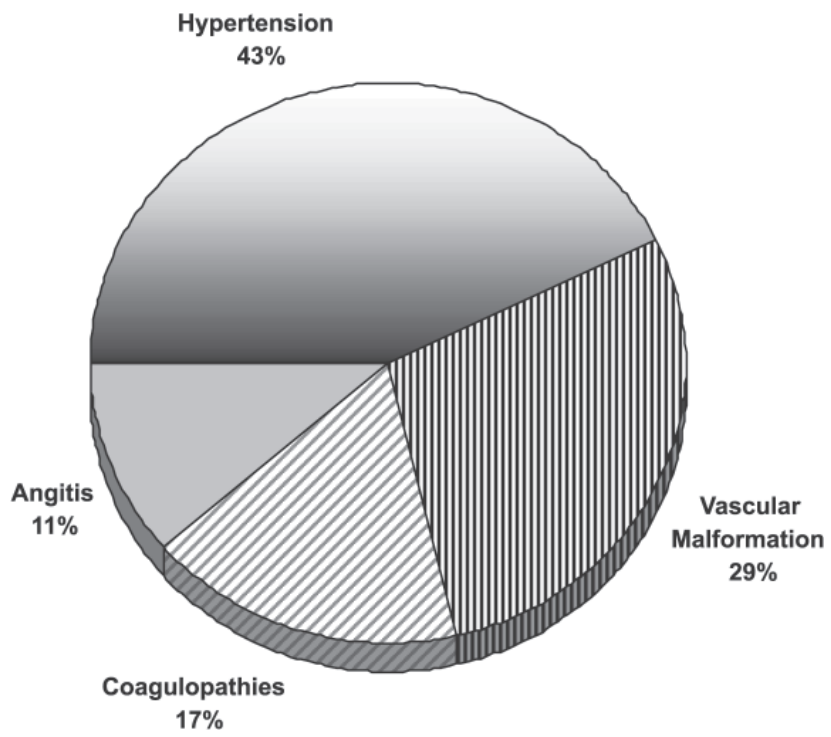

Figure 2 - Causes of Hemorrhagic Stroke in Young People. cerebellum was the site of the lesion. In four $(22 \%)$ cadavers, the neuropathological findings were represented by lobar hematoma secondary to one of the following causes: vasculitis, disseminated intravascular coagulation or ruptured vascular malformation. Subarachnoid hemorrhage was present in nine cadavers $(25 \%)$, seven of these were the result of a ruptured aneurysm and two were the product of a ruptured arteriovenous malformation. Hypertensive cardiopathy was diagnosed in all cases of subarachnoid hemorrhage secondary to the rupture of a sacular aneurysm, whereas chronic renal disease was seen in three cases (42.8\%). Multiple petechiae were found in seven cases $(19.4 \%)$; these were secondary to vasculitis, leukemia-associated blood dyscrasias and idiopathic thrombocytopenic purpura. Intraventricular hemorrhage was seen in two cases $(5.5 \%)$. As for the hemorrhagic stroke group, chronic pyelonephritis was observed in five cadavers $(13.8 \%)$ and nephrosclerosis in nine $(25 \%)$. Causes of death for the hemorrhagic stroke group were a consequence of cerebral herniation in 26 cases (72\%), pulmonary complications represented the cause of death in four patients $(11.1 \%)$, sepsis in one $(2.7 \%)$ and other causes in five (14\%).

\section{DISCUSSION}

The high frequency of hypertensive cardiopathy and chronic nephropathy related to arterial hypertension is the most significant result in the present series. The possible association between cardiovascular disease and chronic nephropathy strongly suggests a relationship in the early and asymptomatic phases of these pathological entities that eventually lead to the development of stroke. This possibility was suggested by McDonald et al. ${ }^{4}$ who claimed that both pathological processes interact synergistically in a direct or indirect manner, and contribute to the genesis of a significant number of cases of stroke in young adults. We believe that these observations offer a possible explanation of the etiopathogenic mechanisms in the majority of cases of stroke in young adults.

In the present series, most ischemic stroke cases were related to atherosclerotic occlusive disease, cardioembolism and lacunar infarctions. In a significant number of cases, hemorrhagic stroke was linked to hypertensive disease and rupture of intracranial aneurysms. Our findings are similar to those documented in several clinical reports of stroke in young adults ${ }^{5-14}$. Nevertheless, those investigations do not consider the possible influence of renal pathology, or even describe its presence.

Arterial hypertension was conspicuously present in the group of patients with hemorrhagic stroke. The most prevalent associated renal diseases in this group were nephro- 
sclerosis and chronic pyelonephritis. In several studies, arterial hypertension has been considered a main risk factor for stroke in young adults, the same as in elderly patients 6-8,10-12,14. Chester et al. ${ }^{15}$ described nephrosclerosis-like alterations in the kidneys of patients with malignant hypertension-related encephalopathy, and also in patients with non-malignant hypertension. However, no previous clinicpathologic correlation study highlights renal lesions in cases of stroke in young adults.

It is a well known fact that essential arterial hypertension is very common, presumably secondary to a group of heterogeneous causes, although, several observations have suggested that kidney disease participates in its genesis ${ }^{16}$. Hypertensive cardiopathy is a well-recognized risk factor for stroke ${ }^{17}$. Chronic renal diseases are known to represent a cardiovascular risk factor, and an elevated serum creatinine concentration is considered a marker of increased risk for stroke ${ }^{18-20}$. Furthermore, nephrosclerosis is thought to be an expression of a systemic blood vessel pathology that quite often acts upon other vascular beds, such as cardiac and cerebral beds ${ }^{22-23}$. Stroke prevalence is higher in hypertensive patients with nephrosclerosis than in hypertensive patients without nephrosclerosis ${ }^{21}$. Furthermore, nephrosclerosis may begin early in life ${ }^{21}$. This has been observed both in experimental models and in humans without hypertension ${ }^{24}$. These facts could be held accountable for the high frequency of hypertension and nephrosclerosis in this group of young adults with hemorrhagic stroke. Hypertensive cardiopathy was found in all of the cases of subarachnoid hemorrhage following a ruptured intracranial aneurysm, and $42.8 \%$ of these patients presented with concomitant chronic nephropathy. These findings indicate that hypertension is a risk factor for subarachnoid hemorrhage, as previously documented in two different epidemiological studies ${ }^{25-26}$.

The presence of chronic pyelonephritis in $43.7 \%$ of the cases of ischemic stroke in the present series is remarkable, as is the previous history of acute myocardial infarction in $31.2 \%$ of the patients. This constitutes a significant difference with the hemorrhage stroke group (see Table 1). These findings represent signs of a chronic and systemic inflammatory state. Smoking and chronic infection are considered as major sources of systemic inflammation ${ }^{27}$. Chronic infections appear to be linked to a prothrombotic state and thrombus formation, as these conditions augment the risk of cardioembolism and atherothrombotic stroke ${ }^{28}$. As shown in Figure 1, atherosclerotic occlusive disease and lacunar infarctions played a fundamental role in $62 \%$ of the cases of ischemic stroke. It is a well-established fact that atherosclerosis contributes to a high percentage of cases of stroke. At present, atherosclerosis is perceived as a chronic inflammatory vascular condition ${ }^{29}$, and infectious diseases are believed to contribute to its pathophysiology $28,30,31$. Potential mechanisms whereby chronic infections may play a role in atherosclerosis are numerous ${ }^{32}$. Elkind et al., ${ }^{33}$ reported that levels of high-sensitive C-reactive protein are higher in patients over 40 years old with ischemic stroke than in stroke-free subjects. Linsberg and Grau ${ }^{28}$ state that there is no specific link between any chronic infectious disease and stroke; however, these chronic infections may represent risk factors that act in cooperation with other conventional risk factors. In opposition, Markus et al. ${ }^{27}$ are of the opinion that inflammation and chronic infection play a causal role in cardiovascular disease. Moreover, chronic pyelonephritis is associated with hypertension in a significant number of cases ${ }^{34-37}$, and it is likely that it begins in early infancy in an insidious manner ${ }^{34}$. In addition, in a study by Markus et al., ${ }^{27}$ recurrent/chronic urinary infection was demonstrated to represent the third most frequent concurrent chronic infection. Furthermore, van de Graaff et al. ${ }^{38}$ postulate that inflammatory responses induce a thrombogenic state that may be responsible for the increased risk of early ischemic strokes in patients with acute myocardial infarction. For these reasons we consider that in the cases of young adults with ischemic stroke analyzed in this study, chronic pyelonephritis and previous acute myocardial infarction are very likely to be associated with and contribute in a synergistic manner to the pathophysiological mechanisms of stroke by means of inducing a systemic, chronic inflammatory state that acts in conjunction with the intrinsic chronic inflammatory vascular condition of atherosclerosis, and the relationship between the latter and arterial hypertension.

According to our data, stroke in people 18 to 49 years of age represents a subgroup of stroke patients of relatively high prevalence $(21 \%)$ and a rather high mortality rate (36\%). Apparently, in the majority of our cases, hypertensive cardiopathy, premature occlusive atherosclerotic disease, arteriolosclerosis and chronic nephropathy interact directly or indirectly beginning in the asymptomatic phases of these pathological processes. Chronic pyelonephritis appears to represent a significant pathology in the acceleration of the process of arterial hypertension and occlusive atherosclerotic disease in patients with ischemic stroke, whereas hypertension and nephrosclerosis constitute major factors in hemorrhagic stroke cases. However, a possible limitation of this study is its retrospective-observational character. For these reasons, further studies are needed in order to confirm these statements.

The potential intimate association between cardiovascular and renal pathological processes has imperative implications for public health programs that aim to diminish 
the risk of stroke. In addition, the control of arterial hypertension as well as the diagnosis and treatment of chronic infections and renal ailments, which are mandatory for the prevention or reduction of the occurrence of stroke, are of similar value. In attention to the relative high prevalence and mortality rate of stroke in people of ages 18 to 49 years, both preventive actions will lead to a minor incapability rate and improved life expectancy.

\section{REFERENCES}

1. Lammie GA. Hypertensive cerebral small vessel disease and stroke. Brain Pathol. 2002;12:358-370.

2. Salud Mundial:Retos Actuales. Organización Mundial de la Salud 2003. In:Informe sobre la Salud en el Mundo. Forjemos el Futuro. OMS, 2003:19.

3. Maggio EM, Montemor-Netto MR, Gasparetto EL, et al. Doenças cerebrovasculares em pacientes entre 15 e 40 anos. Achados neuropatologicos em 47 casos. Arq Neuropsiquiatr. 2001;59:920-925.

4. McDonald SP, Maguire GP, Hoy WE. Renal function and cardiovascular risk markers in a remote Australian aboriginal community. Nephrol Dial Transplant. 2003;18:1555-1561.

5. Boudouresques G, Hauw JJ, Escourolle R. 318 cases of intracerebral haemorrhage. A pathologic study. Rev Neurol. 1980;135:845-865.

6. Bevan H, Sharma K, Bradley W. Stroke in young adults. Stroke. 1990;21:382-386

7. Martinez-Menendez B, Perez-Sampere A, Cabeza-Alvarez CI, et al Non-traumatic cerebral hecatomb in young adults: A study of 42 cases. Med Clint (Bark). 1991;97:726-728.

8. Del Brutto OH, Sanchez J, Campos X, et al. Non-traumatic intracerebral haemorrhage in young adults living in Guayaquil, Ecuador (South America) Analysis of 151 patients. Funct Neurol. 1999;14:21-28.

9. Yonemura K, Kimura K, Hasegawa Y, et al. Analysis of ischemic stroke in patients aged up to 50 years. Rinsho Shinkeigaku. 2000;40:881-886.

10. Flumignan VH, Matos E, Ferreira $\mathrm{CH}$, et al. Acidente vascular cerebral em pacientes jovens. Analise de 164 casos. Arq Neuropsiquiatr. 2001;59:740-745

11. Leno C, Berciano J, Combarros O, et al. Etiologic study of stroke in 95 young adults. Neurologia. 1995;10:283-287.

12. Mehndiratta MM, Agarwal P, Sen K, et al. Stroke in young adult:A study from university hospital in north India. Med Sci Monit. 2004;10:535541

13. Lee TH, Hsu WC, Chen CJ, et al. Etiologic study of young ischemic stroke in Taiwan. Stroke. 2002;33:1950-1955.

14. Moussa R, Harb A, Menassa L, et al. Etiologic spectrum of intracerebral haemorrhage in young patients. Neurochirurgie. 2006;52:105-109.

15. Chester EM, Agamanolis DP, Banker BQ, et al. Hypertensive encephalopathy: A clinicopathologic study of 20 cases. Neurology. 1978;28:928-939.
16. Keller G, Zimmer G, Mall G, et al. Nephron number in patients with primary hypertension. N Engl J Med. 2003;348:101-108.

17. Verdecchia P, Porcellati C, Reboldi G, et al. Left ventricular hypertrophy as an independent predictor of acute cerebrovascular events in essential hypertension. Circulation. 2001;104:2039-2044.

18. Wannamethee SG, Shaper AG, Perry IJ. Serum creatinine concentration and risk of cardiovascular disease:A possible marker for increased risk of stroke. Stroke. 1997;28:557-563.

19. Garcia de Vinuesa S, Gomez F, Goicoechea MA, et al. Riesgo de enfermedad cardiovascular asociado a la enfermedad renal crónica. Prevalencia y grado de control de los factores de riesgo clásicos. Nefrologia. 2005;25:18-22.

20. Skulk T, Mysliwiec M. Influence of early stage of chronic renal disease on risk of cardiac disease and stroke. Przegl Lek. 2006;63:85-87.

21. Gorostidi M, Marin R. Nefropatia vascular. Concepto y significado. Epidemiologia. Hipertension arterial esencial e insuficiencia renal. Nefroesclerosis. Nefropatia isquémica o aterosclerotica. Nefrologia. 2004;24:73-83.

22. Zoccali C, Mallamaci F, Finocchiaro P. Atherosclerotic renal artery stenosis: Epidemiology, cardiovascular outcomes, and clinical prediction rules. J Am Soc Nephrol. 2002;13:S179-S183.

23. Burchfiel CM, Tracy RE, Chyou PH, et al. Cardiovascular risk factors and hyalinization of renal arterioles at autopsy. The Honolulu Heart Program. Atheros Thromb Vasc Biol. 1997;17:760-768.

24. Myrier A, Hill GS, Simon P. Ischemic renal disease:new insights into old entities. Kidney Int. 1998;54:2-13.

25. Knekt P, Reunanen A, Aho K, et al. Risk factors for subarachnoid haemorrhage in a longitudinal population study. J Clin Epidemiol. 1991;44:933-999.

26. Kim HC, Nam CM, Jee SH, et al. Comparison of blood pressureassociated risk of intracerebral haemorrhage and subarachnoid haemorrhage: Korea Medical Insurance Corporation Study. Hypertension. 2005;46:393-397.

27. Markus HS, Labrum R, Bevan S, et al. Genetic and acquired inflammatory conditions are synergistically associated with early carotid atherosclerosis. Stroke. 2006;37:2253-2259.

28. Lindsberg PJ, Grau AJ. Inflammation and infections as risk factors for ischemic stroke. Stroke. 2003;34:2518-2532.

29. Ross R. Atherosclerosis:an inflammatory disease. N Engl J Med. 1999;340:115-126 
30. Kiechl S, Egger G, Mayr M, et al. Chronic infections and the risk of carotid atherosclerosis: Prospective results from a large population study. Circulation. 2001;103:1064-1070.

31. Paganini-Hill A, Lozano E, Fischberg G, et al. Infection and risk of ischemic stroke. Differences among stroke subtypes. Stroke. 2003;34:452-457.

32. Corrado E, Novo S. Role of inflammation and infection in vascular disease. Acta Chir Belg. 2005;105:567-579.

33. Elkind MSV, Coates K, Tai W, et al. Levels of acute phase remain stable after ischemic stroke. BMC Neurology 2006;6:37. doi:10.1186/14712377-6-37.

34. Arze RS, Ramos JM, Owen JP, et al. The natural history of chronic pyelonephritis in adult. Q J Med. 1982;51:396-410.
35. Kes P, Samoscanec S, Klancir S, et al.. Prevalence of arterial hypertension in patients with radiologic sings of chronic pyelonephritis. Z Urol Nephrol. 1986;79:473-480.

36. Goodship THJ, Stoddart JT, Geetha MD, et al. Long-term follow-up of patients presenting to adult nephrologists with chronic pyelonephritis and "normal" renal function. Q J Med. 2000;93:799-803.

37. de Magalhaes R, Menegon F, de Almeida AR, et al. Development of hypertension in a pyelonephritis-induced model: The effect of salt intake and inability of renal sodium handling. Ren Fail. 2006;28:501-507.

38. van de Graaff E, Dutta M, Das P, et al. Early coronary revascularization diminishes the risk of ischemic stroke with acute myocardial infarction. Stroke. 2006;37:2546-2551. 